\title{
Effect of moderate intakes of different tea catechins and caffeine on acute measures of energy metabolism under sedentary conditions
}

\author{
Nikolaj T. Gregersen ${ }^{1}$, Christian Bitz ${ }^{1}$, Inger Krog-Mikkelsen ${ }^{1}$, Ole Hels ${ }^{2}$, Eva M. R. Kovacs ${ }^{3}$, \\ Jane A. Rycroft ${ }^{4}$, Erik Frandsen ${ }^{5}$, David J. Mela ${ }^{6}$ and Arne Astrup ${ }^{1}$ \\ ${ }^{1}$ Department of Human Nutrition, Faculty of Life Sciences, University of Copenhagen, Rolighedsvej 30, DK-1958 \\ Frederiksberg C, Denmark \\ ${ }^{2}$ National Food Institute, Technical University of Denmark, Sфborg, Denmark \\ ${ }^{3}$ Unilever North America, Englewood Cliffs, NJ, USA \\ ${ }^{4}$ Lipton Institute of Tea, Unilever R\&D Colworth Park, Sharnbrook, Bedfordshire, UK \\ ${ }^{5}$ Department of Clinical Physiology and Nuclear Medicine, Glostrup University Hospital, Glostrup, Denmark \\ ${ }^{6}$ Unilever $R \& D$ Vlaardingen, Vlaardingen, The Netherlands \\ (Received 14 October 2008 - Revised 18 March 2009 - Accepted 7 April 2009 - First published online 18 May 2009)
}

\begin{abstract}
Green tea may stimulate energy metabolism; however, it is unclear if acute effects are caused by specific catechins, caffeine or their combination. The objective of the present study was to examine the separate and combined effects of different catechins and caffeine on energy expenditure (EE) and fat oxidation over a single day. Fifteen healthy, normal-weight males received capsules containing placebo, caffeine alone (150 mg), or caffeine plus a catechin mixture $(600 \mathrm{mg}$ ) enriched in either epigallocatechin-3-gallate (EGCG), epigallocatechin or a mix of catechins, in a randomised cross-over double-blinded design. On each test day EE, respiratory quotient (RQ) and substrate oxidation were measured under sedentary conditions in a respiratory chamber for $13.5 \mathrm{~h}$. We found no significant treatment effect on EE $(P=0 \cdot 20)$ or RQ $(P=0.68)$. EGCG with caffeine insignificantly raised $\mathrm{EE}$ and fat oxidation $v$. caffeine-only and placebo (EE 5.71 (SE 0.12) v. 5.68 (SE 0.14) v. 5.59 (SE 0.13 ) $\mathrm{MJ} / 12.5 \mathrm{~h}$, respectively; fat oxidation 84.8 (SE 5.2) v. 80.7 (SE 4.7) v. 76.8 (SE 4.0) g/12.5 h). Catechin/caffeine combinations at these dosages and mode of application had non-significant acute effects on EE and fat oxidation. The maximum observed effect on EE of about $2 \%$ could still be meaningful for energy balance over much longer period of exposure. However, higher short-term effects reported in the literature may reflect variations in green tea extracts, added caffeine, or synergies with physical activity. The specific mechanisms and conditions that may underpin observed longer-term benefits of catechin-enriched green tea consumption on body composition remain to be confirmed.
\end{abstract}

Green tea catechins: Caffeine: Energy expenditure: Substrate oxidation

The focus on bioactive food ingredients and their potential role in preventing weight gain has increased along with the worldwide increasing obesity epidemic. Obesity develops due to a daily positive energy balance, which in itself may be very small (for example, up to about $200 \mathrm{~kJ} / \mathrm{d}$ ), but over the course of months or years can amount to several kilograms of fat ${ }^{(1)}$. One implication of this is that an increase in energy expenditure (EE) by similar amounts $(200 \mathrm{~kJ} / \mathrm{d})$ could help to prevent such a weight gain, provided that these small effects can be sustained. Several bioactive food ingredients, such as capsaicin (hot peppers), caffeine (coffee, tea, etc) and catechins (green tea) have been suggested to be capable of eliciting an increase in diet-induced thermogenesis, and thereby daily EE; however, the size and consistency of the effects observed with these different ingredients vary ${ }^{(2,3)}$.

Green tea is made from leaves from the Camellia sinensis L. species of the Theaceae family. Green tea is the non-oxidised product, as opposed to oolong and black tea, which are partially and fully oxidised, respectively ${ }^{(2)}$. Therefore, green tea contains larger amounts of catechin polyphenols (catechins) than either oolong or black tea ${ }^{(2,3)}$. Nevertheless, the levels of individual catechins plus the total amount of catechins and caffeine varies considerably between different green tea samples ${ }^{(4,5)}$. However, the most abundant of the catechins in green tea are epicatechin, epigallocatechin (EGC), epicatechin-3-gallate and epigallocatechin-3-gallate (EGCG), the latter being most abundant ${ }^{(2)}$. These catechins are known to differ in bioavailability and pharmacokinetics, with particularly striking differences between EGCG and the de-gallated $\mathrm{EGC}^{(6-8)}$. Furthermore, the bioavailability depends on the form of administration, with greater bioavailability when administered as a supplement in capsules as compared with tea ${ }^{(6)}$.

Abbreviations: ANCOVA, analysis of covariance; EE, energy expenditure; EGC, epigallocatechin; EGCG, epigallocatechin-3-gallate; HR, heart rate; RQ, respiratory quotient; SPA, spontaneous physical activity; VAS, visual analogue scale.

* Corresponding author: Dr Nikolaj T. Gregersen, fax +45 35332483, email ntg@life.ku.dk 
Both catechins and caffeine are believed to be responsible for the acute effects of green tea on EE/thermogenesis and fat oxidation observed in previous studies ${ }^{(9,10)}$. The mechanisms behind the effect of green tea components on thermogenesis and EE are not clearly established, but it is believed that sympathetic nervous system stimulation plays a central role ${ }^{(9,11)}$.

Caffeine has been suggested to elicit its effects by inhibiting the enzyme phosphodiesterase, which degrades intracellular cyclic AMP and further by antagonising adenosine receptors $^{(12)}$. However, the effect of green tea extracts on acute energy metabolism is probably not solely attributable to caffeine $^{(9)}$. The catechins are also believed to be capable of increasing thermogenesis through inhibition of the enzyme catechol- $O$-methyltransferase that degrades noradrenaline ${ }^{(13)}$. Thus, the effects of caffeine and catechins may synergistically increase and prolong the effects of noradrenaline on adrenoreceptors and thereby increase thermogenesis ${ }^{(3,9,14)}$. More recently, Venables et al. ${ }^{(15)}$ have reported that a catechinrich green tea extract markedly increased fat oxidation during moderate-intensity activity, a situation of elevated adrenergic stimulation. However, not all catechins may be equally effective in affecting energy metabolism. EGCG in particular has been suggested to have beneficial effects on energy balance and substrate oxidation ${ }^{(16)}$. Furthermore, apart from the potential effects on thermogenesis/EE, catechins and caffeine may decrease appetite and ad libitum energy intake although the current evidence is sparse ${ }^{(17)}$.

In summary, although there seems to be an acute effect of green tea on energy metabolism, the actual size of the effect beyond that of caffeine alone, and which of the catechins generate the greatest effects still need to be clarified. Thus, the primary aim of the present study was to examine the separate and combined effects of green tea extracts enriched in specific catechins $v$. caffeine alone on energy metabolism and fat oxidation over a single day. A secondary objective was to assess the effects of these extracts on subjective appetite sensations.

\section{Methods}

\section{Subjects}

Based on previous results ${ }^{(18)}$ and a power of $0 \cdot 8$, we calculated that fifteen subjects were sufficient to detect a $250 \mathrm{~kJ} / \mathrm{d}(2 \%)$ increase in daytime EE. Therefore, sixteen healthy normalweight male subjects recruited from universities in Copenhagen and Internet advertisements were included in the study. Smokers and elite athletes were excluded from participation, as were individuals with habitual caffeine and catechins intakes above 250 or $200 \mathrm{mg} / \mathrm{d}$, respectively, as well as users of any dietary supplements including vitamins during and from 3 months before the study. Before entering the study, the subjects were screened for blood pressure, body weight and height. All the subjects signed a written informed consent before participating in the study, which was in accordance with the Helsinki Declaration and approved by The Municipal Ethical Committee of Copenhagen and Frederiksberg (KF 01279421). The study was registered at ClinicalTrials.gov (ClinicalTrials.gov no. NCT00611416).

One subject dropped out of the study, because he was unable to take part in the remaining test days for unknown reasons. Thus, fifteen subjects completed the study, with a mean age of 23.6 (SD 2.7) years and mean BMI of 22.4 (SD 1.6$) \mathrm{kg} / \mathrm{m}^{2}$ at the first visit.

\section{Experimental design}

The study was a five-way cross-over double-blinded placebo-controlled design. At each test day, EE, respiratory quotient (RQ) and substrate oxidations were measured via indirect calorimetry for $13.5 \mathrm{~h}$ in one of two whole-body respiratory chambers, and calculated using the formulas of Elia \& Livesey ${ }^{(19)}$. Before the chamber stays, body composition was determined by dual-energy X-ray absorptiometry (DXA) scanning (GE Lunar Prodigy Advance; GE Medical Systems, Lunar, Madison, WI, USA). The measurements of fat-free mass and fat mass were used to adjust EE for body composition. The validity of the DXA scans has previously been described ${ }^{(20,21)}$.

RMR was measured before the test days using ventilated hoods. (The precision of the ventilated hood system was validated on a weekly basis by an alcohol-burning test; CV was $1.5 \%$.) The individually expected $24 \mathrm{~h}$ EE during the chamber stay was calculated by multiplying the measured RMR with a physical activity level factor of 1.4 . The energy intake required to obtain energy balance during the chamber stay was predicted from the calculated $24 \mathrm{~h}$ EE value. However, since EE was only measured for $13.5 \mathrm{~h}$, this was not a balance study. Therefore, we report treatment effects on EE, RQ and substrate oxidation, and not overall energy or macronutrient balances.

\section{Treatments}

The five different treatments in the present study were given as capsules, and the treatments and the content of these capsules are described in Table 1. The capsules were

Table 1. Content of the green tea capsules $(\mathrm{mg})$

\begin{tabular}{|c|c|c|c|c|c|c|c|c|c|c|}
\hline Treatment & $\begin{array}{c}\text { Caffeine } \\
\text { (per capsule) }\end{array}$ & C & EC & ECG & EGC & EGCG & $\mathrm{GC}$ & GCG & $\begin{array}{l}\text { Total catechins } \\
\text { (per capsule) }\end{array}$ & $\begin{array}{c}\text { Total catechins } \\
\text { (per d) }\end{array}$ \\
\hline Placebo & 0 & 0 & 0 & 0 & 0 & 0 & 0 & 0 & 0 & 0 \\
\hline EGCG and caffeine & $25 \cdot 0$ & 0.2 & 0.9 & $4 \cdot 2$ & 0.2 & $101 \cdot 2$ & 0 & 0.8 & $107 \cdot 5$ & $645 \cdot 0$ \\
\hline EGC and caffeine & $25 \cdot 0$ & 0.6 & $7 \cdot 5$ & 0 & $95 \cdot 3$ & 0.3 & $10 \cdot 3$ & 0 & 114.0 & 684.0 \\
\hline Catechins and caffeine & $25 \cdot 0$ & $1 \cdot 0$ & $7 \cdot 5$ & $7 \cdot 1$ & $20 \cdot 5$ & $40 \cdot 4$ & $3 \cdot 3$ & 2.4 & $82 \cdot 3$ & $493 \cdot 8$ \\
\hline Caffeine & $25 \cdot 0$ & 0 & 0 & 0 & 0 & 0.1 & 0 & 0 & 0.1 & 0.6 \\
\hline
\end{tabular}

C, catechin; EC, epicatechin; ECG, epicatechin-3-gallate; EGC, epigallocatechin; EGCG, epigallocatechin-3-gallate; GC, gallocatechin; GCG, gallocatechin gallate. 
developed and standardised by Unilever Research \& Development Colworth (Sharnbrook, Beds, UK). The daily levels of catechins in the active treatments (493.8-684.0 mg) correspond to approximately the catechin content of three cups of green tea ${ }^{(4)}$. Likewise, the daily amount of caffeine in the active treatments $(150 \mathrm{mg})$ is equivalent to the amounts of caffeine in either three cups of green tea or a cup of espresso ${ }^{(4,22)}$.

\section{Standardisation}

Subjects were asked to refrain from exercise and eliminate consumption of foods or beverages containing caffeine (coffee, tea, cola, etc) and catechins (chocolate, red wine, apples and pears) for $24 \mathrm{~h}$ before each chamber stay. The subjects received meals (prepared at the research site) for the entire day before each test day, and during this day they were only allowed to drink water. The standardised dinner meal (50\% energy as carbohydrate, $37 \%$ energy as fat and $13 \%$ energy protein) was to be eaten before 20.00 hours of the evening before the test days. From 20.00 hours the evening before the test day until the beginning of the test day, the subjects were only allowed to drink 0.5 litres of tap water. Subjects were measured in the same respiratory chamber on each of the five visits in order to minimise the within-subject variation.

\section{Protocol}

The test day started at 07.30 hours with subjects arriving at the department. After voiding of the bladder and subsequent weighing, a mobile digital pulse oximetry device was attached to the subject (Nonin Avant 4000; Nonin Medical Inc., Plymouth, MN, USA) to allow for continuous heart rate (HR) measurements during the chamber stay. The subject then entered the respiratory chamber. At 08.00 hours, the chamber was sealed, and subjects then rested on the bed for $30 \mathrm{~min}$. At 08.30 hours, measurements of $\mathrm{EE}$ and RQ were begun, and the first hour was dedicated to measuring RMR. Standardised meals were given at 09.45 hours (breakfast; $23 \%$ daily energy intake), 13.45 hours (lunch; $33 \%$ daily energy intake), 17.45 hours (dinner; $36 \%$ daily energy intake) and 21.30 hours (snack; $8 \%$ daily energy intake). All the meals were prepared by a specially educated kitchen staff using standardised recipes. The six capsules were given at 09.30 hours (two capsules), 11.45 hours (one capsule), 13.30 hours (one capsule), 15.45 hours (one capsule) and 17.30 hours (one capsule). At 22.00 hours the subjects were let out of the chamber and the test day was over; no further food intake was allowed until 08.00 hours next morning.

During the chamber stay (08.00-22.00 hours) and the following night (22.00-08.00 hours) the subjects collected all urine in plastic containers containing $25 \mathrm{ml}$ of a $10 \%$ $\mathrm{HCl}$ solution. The volume of the urine samples was measured, and small representative samples were then drawn from the larger samples and stored at $-80^{\circ} \mathrm{C}$. The $\mathrm{N}$ content was measured using a nitrogen analyser (Carlo Erba Strumentazione NA 1500, 1986; Thermo Fisher Scientific, Slangerup, Denmark). Adrenaline and noradrenaline were measured by RIA using a commercial kit (2-Cat RIA; LDN, Nordhorn, Germany). Spontaneous physical activity (SPA) in the respiratory chamber was measured by IR detectors (Minilux Sensor
PIR 41-162; Servodan A/S, Sønderborg, Denmark). SPA is expressed as the percentage of time in which the subject has a detectable level of physical activity.

\section{Subjective appetite sensations}

Visual analogue scales (VAS) were used to determine changes in subjective appetite sensations during the chamber stay. Ten different appetite sensations were examined. These were: satiety, hunger, fullness, prospective food consumption, thirst, wellbeing, and desire to eat something fatty, sweet, salty and savoury. The subjects filled out the VAS after $0(08.00$ hours), 90, 120, 180, 240, 300, 330, 360, 420, 480, 540, $570,600,660,720$ and $780 \mathrm{~min}$. The use, reproducibility and validity of the VAS have been described in a previous report from this laboratory ${ }^{(23)}$. However, because the trial was powered for the energy metabolism measures, and appetite effects of green tea components are not well known, we analysed these as a secondary outcome.

\section{Data}

No measurements were made during the first $30 \mathrm{~min}$ of the chamber stay (from 08.00-08.30 hours). During the following $30 \mathrm{~min}$ period of the chamber measurements (08.30-09.00 hours), the $\mathrm{O}_{2}$ analyser did not reach equilibrium and thus produced invalid results. This $30 \mathrm{~min}$ period was therefore excluded and the measurements from the $30 \mathrm{~min}$ period between 09.00 and 09.30 hours were used for calculating RMR. Thus, the statistical analyses were based on data from the remaining $12.5 \mathrm{~h}$ period (9.30-22.00 hours), and therefore both RQ and EE measures refer to $12.5 \mathrm{~h}$ data unless otherwise stated.

Given the design of the study with dosing throughout the day, the acute effects of the green tea capsules were also examined by analyses of the data divided into specific time blocks. This was in order to examine whether there were any immediate or acute effects of the active treatments in the first few hours after each capsule that may not have been visible over the duration of an entire day. The time blocks were 09.30-11.45 hours (block 1), 11.45-15.45 hours (block 2) and 15.45-22.00 hours (block 3).

Incremental areas under the curve were calculated for the VAS scores for satiety and fullness, and incremental areas over the curve were calculated for the VAS scores for hunger and prospective food consumption.

\section{Statistical analyses}

Data were analysed by mixed-model analysis of covariance (ANCOVA). RQ, EE, HR, fat and carbohydrate oxidations were evaluated as independent variables. Treatment, chamber, period (visit number) and interaction between treatment and period were evaluated as independent fixed variables. Age, body weight, fat-free mass, fat mass, energy intake during chamber stay and SPA were evaluated as covariates and subjects as a dependent random variable. Where the main effect of treatment was significant, post hoc comparisons were made using $t$ tests with Tukey-Kramer adjustment of significance levels for the pair-wise comparisons of treatments. 
Furthermore, repeated-measures ANCOVA were performed in order to examine differences in time within periods. In these models, subjects were nested within periods.

In order to adjust for differences in SPA between the two respiratory chambers we conducted repeated-measures ANCOVA with EE, RQ and HR as independent variables, and treatment, period, time as well as two-factor interactions as dependent fixed variables. Subjects were included as a dependent random variable and SPA as a covariate.

The data on SPA were analysed by ANCOVA with SPA level as an independent variable, whereas treatment, period and interaction between treatment and period were evaluated as independent fixed variables. Age, body weight, fat-free mass and fat mass were evaluated as covariates and subjects as a dependent random variable.

The catecholamine data were analysed by ANCOVA with $24 \mathrm{~h}$ adrenaline and noradrenaline levels as independent variables, whereas treatment, period and interaction between treatment and period were evaluated as independent fixed variables. Age and body weight were evaluated as covariates and subjects as a dependent random variable.

VAS scores for satiety, hunger, fullness and prospective food consumption were analysed as both summary measures using incremental areas under the curve and incremental areas over the curve, and as repeated measures using a mixed-model ANCOVA. VAS scores for desire to eat something salty, fatty, savoury and sweet, and VAS scores for thirst and wellbeing were only analysed as repeated measures using a mixed-model ANCOVA. Where the main effect of treatment was significant, post hoc comparisons were made with Tukey-Kramer adjustment of significance levels for the pair-wise comparison of treatments. Treatment, period and interaction between treatment and period were evaluated as independent fixed variables. Age and body weight were evaluated as covariates and subjects as a dependent random variable.

In all statistical models, residuals were tested for normality by Shapiro-Wilk tests and variance homogeneity was investigated using residual plots. All results are given as means with their standard errors. The significance level was set at $P<0 \cdot 05$. Statistical analyses were performed using SAS (version 9.1; SAS Institute, Cary, NC, USA).

\section{Results}

\section{Energy expenditure}

The multivariate ANOVA showed no significant overall treatment effect on $\mathrm{EE}(P=0 \cdot 20)$ (Fig. 1). Body weight was a significant covariate $(P=0.0002)$, as would be expected since EE normally correlates with body weight as seen from formulas used to predict $\mathrm{EE}^{(19)}$. Likewise, the repeatedmeasures ANCOVA did not show any effect of treatment on $\mathrm{EE}(P=0 \cdot 84)$, but both time and body weight were significant covariates, which again could be expected due to the association between body weight and $\mathrm{EE}$, and the fact that $\mathrm{EE}$ changes over time due to the intake of meals $(P<0 \cdot 0001)$.

When analysing the data split into the previously mentioned time blocks, we observed no treatment effect on EE in time blocks 1 and 2 , but in time block 3 there was a tendency towards a treatment effect on $\mathrm{EE}(P=0.053)$ (Table 2).

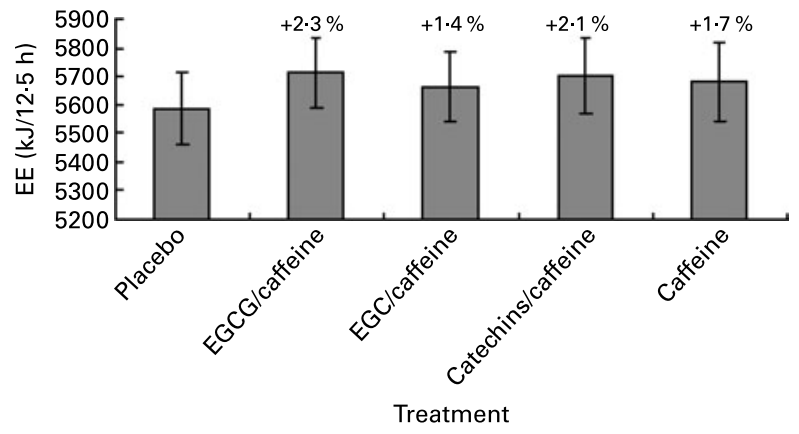

Fig. 1. Energy expenditure (EE) during the $12.5 \mathrm{~h}$ chamber stay plotted against the five different treatments. Differences between single treatments and placebo are given as percentages above each bar. Data are means ( $n$ 15), with standard errors represented by vertical bars. EGCG, epigallocatechin-3-gallate; EGC, epigallocatechin.

Post hoc comparison (Tukey-Kramer) of EE data from time block 3 indicated that the difference between EGCG/caffeine $(0.46 \mathrm{MJ} / \mathrm{h})$ and placebo $(0.44 \mathrm{MJ} / \mathrm{h})$ was borderline statistically significant $(P=0 \cdot 05)$. There was also a trend in EE for time block 3 for caffeine only $(0.46 \mathrm{MJ} / \mathrm{h}) \quad v$. placebo $(0.44 \mathrm{MJ} / \mathrm{h}) \quad(P=0.08)$. However, there was no difference among any of the active treatments.

\section{Respiratory quotient and substrate oxidations}

The multivariate ANOVA showed that there was no overall treatment effect on RQ $(P=0.68)$, but that fat mass was a significant covariate $(P=0.0023)$ (Fig. 2), which could be expected since fat mass has been shown to correlate with fat oxidation in both normal-weight and obese subjects ${ }^{(24)}$. Likewise, repeated-measures ANCOVA indicated no overall effect of treatment on RQ $(P=0.75)$, but again fat mass was a significant covariate $(P=0 \cdot 0078)$. Furthermore, according to mixed-model ANCOVA with these same covariates, there were no overall treatment effects on RQ when analysed as time blocks (Table 2).

Fat oxidation for the five different treatments is shown in Fig. 3. From Fig. $3^{(2)}$ it might appear that fat oxidation with EGCG/caffeine was greater than for the placebo in particular; however, there were no significant treatment effects $(P=0 \cdot 55)$, and also no significant covariates.

Lastly, there were no treatment differences in carbohydrate oxidation during the $12.5 \mathrm{~h}$ period $(P=0.81)$.

Table 2. Results of statistical analyses on time block data

\begin{tabular}{lcll}
\hline Outcome & Time block $\dagger$ & $P$ & Significant covariates \\
\hline EE & 1 & 0.29 & $\mathrm{FFM}^{\star \star}, \mathrm{FM}^{*}$ \\
& 2 & 0.47 & $\mathrm{FFM}^{\star \star}$ \\
$\mathrm{RQ}$ & 3 & 0.053 & $\mathrm{FFM}^{\star \star}$ \\
& 1 & 0.61 & \\
& 2 & 0.64 & $\mathrm{FFM}^{\star}, \mathrm{FM}^{\star *}$ \\
\hline
\end{tabular}

$E E$, energy expenditure; FFM, fat-free mass; $F M$, fat mass; $R Q$, respiratory quotient.

${ }^{\star} P<0.05,{ }^{\star *} P<0.01$.

†Time block 1, 09.30-11.45 hours; time block 2, 11.45-15.45 hours; time block 3 , $15.45-22.00$ hours. 


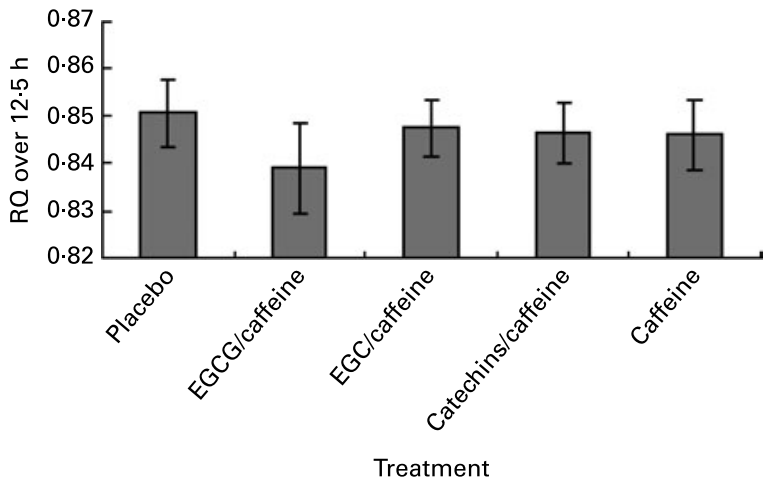

Fig. 2. Respiratory quotient $(R Q)$ during the $12 \cdot 5 \mathrm{~h}$ chamber stay plotted against the five different treatments. Data are means $(n 15)$, with standard errors represented by vertical bars. EGCG, epigallocatechin-3-gallate; EGC, epigallocatechin.

\section{Heart rate}

Neither the multivariate ANOVA nor the repeated-measures ANCOVA showed any treatment effects on $\mathrm{HR}(P=0 \cdot 17$ and $P=0.15$, respectively). However, for the repeatedmeasures ANCOVA, SPA $(P=0.0024)$ and time $(P<0.0001)$ were significant covariates, which could be expected since it is well known that increased SPA increases HR and that HR can vary during the day due to meals and changes in physical activity. When divided into time blocks, we observed no treatment effects on HR for time blocks 1 and $3(P=0.07$ and $P=0.57$, respectively), although there was a trend toward an effect (lower HR in EGCG/caffeine $v$. placebo) in time block 1 . However, for time block 2 we observed a significant treatment effect on HR $(P=0 \cdot 037)$. Subsequent post hoc analyses (Tukey-Kramer) showed that EGCG/caffeine reduced HR compared with placebo $(P=0.032)$, whereas no other treatment differences were observed.

\section{Spontaneous physical activity}

There were small but statistically significant differences in SPA between treatments $(P=0 \cdot 049)$. Post hoc (Tukey-Kramer) analyses showed that SPA was slightly higher during the

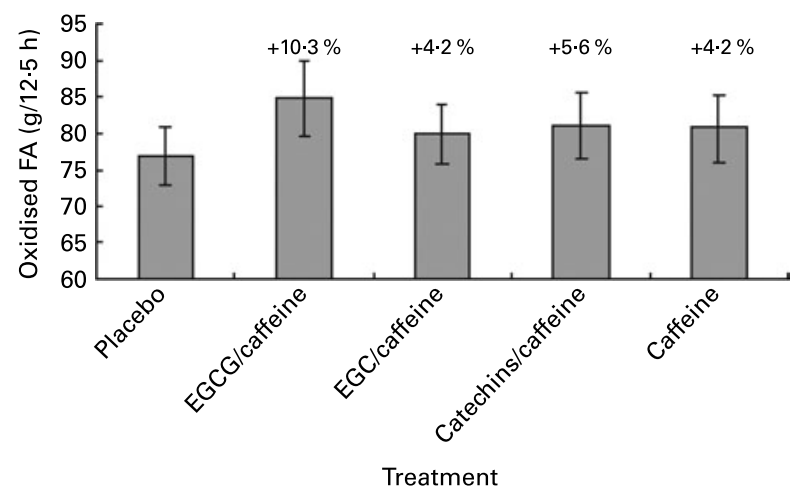

Fig. 3. Fat oxidation during the $12.5 \mathrm{~h}$ chamber stay plotted against the five different treatments. Differences between single treatments and placebo are given as percentages above each bar. Data are means $(n 15)$, with standard errors represented by vertical bars. FA, fatty acids; EGCG, epigallocatechin3-gallate; EGC, epigallocatechin. catechins/caffeine (SPA 13.89\%) treatment compared with the caffeine-only (SPA $11.45 \%)$ treatment $(P=0.0389)$. The SPA during the remaining three treatments (placebo, SPA $13.04 \%$; EGCG/caffeine, SPA $11.97 \%$; EGC/caffeine, SPA $12.54 \%$ ) were not significantly different from any of the other treatments.

\section{Subjective appetite sensations}

VAS ratings of hunger, prospective food consumption and fullness showed no significant differences between treatments $(P>0 \cdot 20)$. There was a tendency for all active treatments to result in larger levels of satiety (incremental areas under the curve) compared with placebo $(P=0 \cdot 055)$.

Furthermore, there was a significant treatment effect on the desire to eat something salty $(P<0 \cdot 01)$. Post hoc TukeyKramer adjusted analyses showed that the caffeine-only treatment resulted in decreased desire for something salty compared with placebo $(P<0.01)$ and similar trends for the EGC/caffeine and catechins/caffeine treatments compared with placebo $(P=0.079$ and $P=0.054$, respectively $)$. Finally, there were no treatment effects on the desire to eat something fatty, savoury or sweet, and likewise no treatment effects on thirst sensations or wellbeing $(P>0 \cdot 13)$.

\section{Catecholamines}

The statistical analyses showed no effects of treatments on $24 \mathrm{~h}$ urine levels of adrenaline $(P=0.28)$ or noradrenaline $(P=0 \cdot 17)$ (Table 3$)$. However, body weight was a significant covariate in both analyses, meaning that the larger the body weight the larger the levels of the catecholamines $(P<0.001$ and $P<0.01$, respectively).

\section{Discussion}

In the present study we found no statistically significant evidence of an acute (within-day) effect of green tea extracts with varying levels of putative active ingredients on EE, RQ or substrate oxidation. There were some non-significant tendencies (Figs. 1-3) towards increased EE $(<2 \%)$ and fat oxidation, and decreased RQ (about $10 \%$ ) on the active treatments (especially EGCG/caffeine) compared with placebo.

These results contrast with the much larger acute effects of green tea extracts reported in some other studies. In particular, Dulloo et al. reported increases in EE by $3.5 \%$ and in fat oxidation $\left(27 \mathrm{~g} / 24 \mathrm{~h}\right.$ increase) with green tea ${ }^{(9)}$. Bérubé-Parent et al. reported an increase in $24 \mathrm{~h} \mathrm{EE}$ by about $750 \mathrm{~kJ}$ on different combinations of catechins and caffeine (from green tea and guarana) ${ }^{(25)}$. Rudelle et al. reported a $4.6 \%$ increase in $24 \mathrm{~h} \mathrm{EE}$ (though no significant changes in macronutrient oxidation) with a green tea extract and caffeine ${ }^{(10)}$. Lastly, Venables et al. reported a $17 \%$ increase in fat oxidation during moderate activity, following green tea extract ${ }^{(15)}$.

Based on previous research, the present study was powered to detect a difference of $250 \mathrm{~kJ}$ (about $2 \%$ ) in EE during the measuring period. Notably, however, effects on EE did not approach statistical significance (overall treatment $P=0 \cdot 20$ ), except in secondary analyses of a single time block (time block 3). The duration of the treatments does not seem to be 
Table 3. Catecholamine concentrations in $24 \mathrm{~h}$ urine samples

(Mean values with their standard errors for fifteen subjects)

\begin{tabular}{|c|c|c|c|c|c|c|c|c|c|c|c|}
\hline \multirow[t]{2}{*}{ Treatment ... } & \multicolumn{2}{|c|}{ Placebo } & \multicolumn{2}{|c|}{$\begin{array}{l}\text { EGCG and } \\
\text { caffeine }\end{array}$} & \multicolumn{2}{|c|}{ EGC and caffeine } & \multicolumn{2}{|c|}{$\begin{array}{l}\text { Catechins and } \\
\text { caffeine }\end{array}$} & \multicolumn{2}{|c|}{ Caffeine } & \multirow[b]{2}{*}{$P$} \\
\hline & Mean & SE & Mean & SE & Mean & SE & Mean & SE & Mean & SE & \\
\hline Adrenaline ( $\mu \mathrm{mol} / 24 \mathrm{~h})$ & 0.0547 & 0.0061 & 0.0473 & 0.0027 & 0.0487 & 0.0050 & 0.0533 & 0.0045 & 0.0527 & 0.0052 & 0.28 \\
\hline Noradrenaline $(\mu \mathrm{mol} / 24 \mathrm{~h})$ & 0.3153 & 0.0286 & 0.2993 & 0.0190 & 0.2993 & 0.0206 & 0.3320 & 0.0205 & 0.3133 & 0.0237 & 0.17 \\
\hline
\end{tabular}

EGCG, epigallocatechin-3-gallate; EGC, epigallocatechin.

responsible for the differences in results between studies, since two of the previous studies ${ }^{(9,25)}$ had similar duration to the present study ( $1 \mathrm{~d}$ with three daily doses), whereas the study by Rudelle et al. ${ }^{(10)}$ had a longer treatment period $(3 \mathrm{~d}$ with three daily doses).

The amounts of catechins used here were also well within the range of other studies. Bérubé-Parent et al. reported no additional dose-response effect on EE with varying daily dosing size of EGCG from 270 to $1200 \mathrm{mg} / \mathrm{d}^{(25)}$ in the presence of $600 \mathrm{mg}$ caffeine. Furthermore, Dulloo et al. ${ }^{(9)}$ saw significant effects on EE with an EGCG level lower than or equal $(270 \mathrm{mg} / \mathrm{d})$ to amounts used both by BérubéParent et al. ${ }^{(25)}$ and the present study. Most recently, Rudelle et al. reported significant effects on EE with a catechin level $(540 \mathrm{mg} / \mathrm{d} ; 50 \%$ of which EGCG) similar to the present study, though with large quantities of added caffeine $(300 \mathrm{mg} / \mathrm{d})^{(10)}$.

To our knowledge, the present study is the first in human subjects to specifically examine whether different catechins differ in their ability to affect EE and substrate oxidation. However, we observed no significant differences between the specific catechins tested with regard to these endpoints. Although the total daily amount of catechins varied between the catechin-containing treatments in the present study (range $493.8-684.0 \mathrm{mg} / \mathrm{d}$ ) it seems unlikely that these differences influenced the present results, since the amounts used in the present study are all within the ranges for which effects have been reported in previous studies ${ }^{(9,10,25)}$. Thus, neither the differences in dosing sizes between treatments in the present study nor the different types of catechins seem to explain the differences in short-term effects seen between the present and the other three studies.

A more likely potential reason for the differences between studies and treatments could be the content of (added) caffeine. In the present study the effect of caffeine per se was tested in addition to the effect of the catechins (and caffeine) from the capsules containing the green tea extracts. Even though the EGCG/caffeine treatment numerically seemed to be the most potent stimulator of $\mathrm{EE}$ and fat oxidation in the present study, the differences between the active treatments were rather small and non-significant, and thus there is a chance that the tendency towards an effect that we found is primarily due to caffeine and not catechins. This would be consistent with findings of Bérubé-Parent et al. where varying the catechin content from 270 to $1200 \mathrm{mg} / \mathrm{d}$, plus $600 \mathrm{mg} / \mathrm{d}$ caffeine, did not influence the size of the effect on $\mathrm{EE}^{(25)}$. Also, Rudelle et al. ${ }^{(10)}$ reported significant effects on $24 \mathrm{~h}$ EE with a level of catechins similar to the present study, but using twice the level of caffeine (300 v. $150 \mathrm{mg} / \mathrm{d})$. However,
Dulloo et al. reported a larger $24 \mathrm{~h} \mathrm{EE}$ and fat oxidation, and a lower RQ on the green tea treatment compared with caffeine alone (similar caffeine contents of $150 \mathrm{mg}$ ), and those authors suggested that the green tea extract resulted in effects beyond those that could be explained by the caffeine content ${ }^{(9)}$. Thus, the study by Dulloo et al. is the only to report acute effects of green tea on EE and fat oxidation, without substantial amounts of added caffeine content ${ }^{(9)}$. A potential explanation for this discrepancy between the present study and the study by Dulloo et al. ${ }^{(9)}$ could be the different modes of dosage. In both studies the daily dose of caffeine was $150 \mathrm{mg}$, but in the present study the dosage was $1 \times 50 \mathrm{mg}+4 \times 25 \mathrm{mg}$, whereas in the Dulloo et al. study it was $3 \times 50 \mathrm{mg}$. Thus, a dosage below $50 \mathrm{mg}$ per administration could be beneath the threshold for a synergistic effect between catechins and caffeine and thereby explain the difference in results. Therefore, there are still some doubts about the actual magnitude of acute effects of catechins per se on energy and lipid metabolism, and the present study seems to suggest that the larger effect sizes reported elsewhere may often be due to or potentiated by higher levels of caffeine or perhaps synergy with physical activity ${ }^{(15,26)}$.

Even though the acute effect $(24 \mathrm{~h})$ of the green tea combinations we examined was smaller than expected (and non-significant) relative to previous research, this does not rule out the possibility that green tea can have beneficial effects on energy balance and body fat stores over longer time periods ${ }^{(26)}$. A number of studies have shown that longer-term consumption of green tea extracts can decrease body weight, waist circumference, total body fat and visceral $\mathrm{fat}^{(27-32)}$, the latter being viewed as a particular health risk $^{(33)}$. However, other studies have failed to find such long-term effects on body weight and composition during ${ }^{(34)}$ or following $^{(35)}$ a low-energy weight-loss diet. It is possible that small effects of green tea are less apparent against the background of a larger change in diet or energy balance. However, in support of the benefits of green tea for weight control, a recent study has observed that $10 \mathrm{~d}$ supplementation with polyphenol-enriched oolong tea can increase fat excretion ${ }^{(36)}$. Thus, this effect may also be partly responsible for the long-term effects of green tea seen in some studies $^{(27-32)}$. Nevertheless, it is not known whether this effect is meaningful and persistent over longer time periods. Thus, further studies are needed to clarify the weight-loss/ weight-maintenance potential of green tea, and particularly the mechanisms behind the effects observed in a number of trials $^{(27-32)}$. In addition, based on the observations that most studies showing effects of green tea catechins are conducted in Asian subjects it seems important to clarify whether the 
effects of green tea catechins differ between different population groups.

The fact that EGCG reduced HR compared with placebo in time block 2 (11.45-15.45 hours) was surprising since one could have expected an increase in HR following the thermogenic EGCG treatment, because of the likely activation of the sympathetic nervous system. However, Hill et al. have previously shown that 12-week EGCG supplementation reduced HR compared with placebo ${ }^{(37)}$. Nevertheless, another study failed to find any effects of EGCG on $\mathrm{HR}^{(34)}$. Furthermore, none of the other statistical analyses showed any treatment effects on HR and, thus, this effect does not seem to be strong and consistent over time.

The level of SPA was slightly larger during the catechins/ caffeine treatment compared with the caffeine-only treatment. This was unexpected and probably incidental, as there is no apparent causal basis for such a result from the present or previous studies. Because SPA was a covariate in analyses of $\mathrm{EE}$ and RQ, differences in SPA levels should not have influenced the interpretation of results for these endpoints.

We observed no effect of treatments on urinary catecholamine excretion. This finding was in agreement with the findings of two other studies ${ }^{(10,25)}$, but contradicts the findings in a third study ${ }^{(9)}$. However, it is consistent with the fact that there were also no effects on $\mathrm{EE}$ and fat oxidation. This suggests that the treatments did not affect the sympathetic nervous system sufficiently to elicit significant effects on the above-mentioned endpoints. However, urinary catecholamine excretion is a crude proxy for sympathetic activity as it reflects the outcome of spillover from the sympathetic nerve endings, degradation and renal excretion.

Lastly, we found no significant effect of the treatments on subjective appetite sensations, and only a weak tendency for an effect on satiety.

\section{Conclusions}

The present results suggest that the tested catechins/caffeine combinations in sedentary conditions at these dosages and with this mode of application exert only small acute effects on $\mathrm{EE}$ and fat oxidation, which were not statistically significant. Positive trends observed indicate that the effect size of catechins/caffeine combinations on EE under these conditions would at most be in the order of about $2 \%$. Such a small level of effect could still contribute toward the longerterm cumulative effects on energy balance observed in many long-term studies; however, the higher acute effects reported in the literature may reflect variations in green tea extracts, or enhancement of responses when combined with added caffeine or physical activity. Furthermore, there does not seem to be an effect of the tested catechins/caffeine combinations on subjective appetite measures. There remains a need to identify the specific mechanisms or their combination, perhaps individually of small magnitude, that might underpin observations of longer-term effects of green tea catechins on energy balance.

\section{Acknowledgements}

The authors gratefully thank Yvonne Rasmussen, Berit Hoielt, Karina Graff Rossen, Kira Holst Bjerre Larsen, Charlotte
Kostecki, Martin Kreutzer, John Lind and Søren Andresen for expert technical assistance, and the subjects for their willing participation. In addition, the authors thank Anita Belza for assistance in designing the study, and Christian Ritz for expert statistical assistance.

C. B, E. M. R. K., J. A. R., D. J. M. and A. A. planned the present study, and C. B. wrote the protocol. C. B. and I. K.-M. conducted the study. N. T. G. analysed the data with O. H., E. M. R. K., J. A. R., D. J. M. and A. A. all providing help. N. T. G. wrote the manuscript, and the remaining authors all reviewed the manuscript.

The present study was supported by the Unilever R\&D Vlaardingen, The Netherlands.

E. M. R. K., J. A. R. and D. J. M. are employees of a company that manufactures tea products.

\section{References}

1. Hill JO, Wyatt HR, Reed GW, et al. (2003) Obesity and the environment: where do we go from here? Sci Tech Froid 299, $853-855$.

2. Kovacs EMR \& Mela DJ (2006) Metabolically active functional food ingredients for weight control. Obes Rev 7, 59-78.

3. Westerterp-Plantenga MS, Diepvens K, Joosen AMCP, et al. (2006) Metabolic effects of spices, teas, and caffeine. Physiol Behav 89, 85-91.

4. Reto M, Figuera ME, Filipe HM, et al. (2007) Chemical composition of green tea (Camellia sinensis) infusions commercialized in Portugal. Plant Foods Hum Nutr 62, 139-144.

5. Jin Y, Jin CH \& Row KH (2006) Separation of catechin compounds from different teas. Biotechnol J 1, 209-213.

6. Van Amelsvoort JMM, van Het Hof KH, Mathot JNJJ, et al. (2001) Plasma concentrations of individual tea catechins after a single oral dose in humans. Xenobiotica 31, 891-901.

7. Henning SM, Niu Y, Lee NH, et al. (2004) Bioavailability and antioxidant activity of tea flavanols after consumption of green tea, black tea, or a green tea extract supplement. Am J Clin Nutr 80, 1558-1564.

8. Warden BA, Smith LS, Beecher GR, et al. (2001) Catechins are bioavailable in men and women drinking black tea throughout the day. J Nutr 131, 1731-1737.

9. Dulloo AG, Duret C, Rohrer D, et al. (1999) Efficacy of a green tea extract rich in catechin polyphenols and caffeine in increasing 24-h energy expenditure and fat oxidation in humans. Am J Clin Nutr 70, 1040-1045.

10. Rudelle S, Ferruzzi MG, Cristiani I, et al. (2007) Effect of a thermogenic beverage on 24-hour energy metabolism in humans. Obesity 15, 349-355.

11. Dulloo AG, Seydoux J, Girardier L, et al. (2000) Green tea and thermogenesis: interactions between catechin-polyphenols, caffeine and sympathetic activity. Int J Obes 24, 252-258.

12. Dulloo AG, Seydoux J \& Girardier L (1992) Potentiation of the thermogenic antiobesity effects of ephedrine by dietary methylxanthines: adenosine antagonism or phosphodiesterase inhibition? Metabolism 41, 1233-1241.

13. Borchardt RT \& Huber JA (1975) Catechol $O$-methyltransferase. 5. Structure-activity relationships for inhibition by flavonoids. J Med Chem 18, 120-122.

14. Belza A, Frandsen E \& Kondrup J (2007) Body fat loss achieved by stimulation of thermogenesis by a combination of bioactive food ingredients: a placebo-controlled, double-blind 8-week intervention in obese subjects. Int J Obes 31, 121-130.

15. Venables MC, Hulston CJ, Cox HR, et al. (2008) Green tea extract ingestion, fat oxidation, and glucose tolerance in healthy humans. Am J Clin Nutr 87, 778-784. 
16. Kao Y-H, Hiipakka RA \& Liao S (2000) Modulation of endocrine systems and food intake by green tea epigallocatechin gallate. Endocrinology 141, 980-987.

17. Belza A, Toubro S \& Astrup A (2009) The effect of caffeine, green tea and tyrosine on thermogenesis and energy intake. Eur J Clin Nutr 63, 57-64.

18. Toubro S, Christensen NJ \& Astrup A (1995) Reproducibility of 24-h energy expenditure, substrate utilization and spontaneous physical activity in obesity measured in a respiration chamber. Int J Obes Relat Metabc Disord 19, 544-549.

19. Elia M \& Livesey G (1992) Energy expenditure and fuel selection in biological systems: the theory and practice of calculations based on indirect calorimetry and tracer methods. World Rev Nutr Diet 70, 68-131.

20. Haarbo J, Godtfredsen A, Hassager C, et al. (1991) Validation of body composition by dual energy X-ray absorptiometry (DEXA). Clin Physiol 11, 331-341.

21. Mazess RB, Barden HS, Bisek JP, et al. (1990) Dual-energy $\mathrm{X}$-ray absorptiometry for total-body and regional bone-mineral and soft-tissue composition. Am J Clin Nutr 51, 1106-1112.

22. Desbrow B, Hughes R, Leveritt M, et al. (2007) An examination of consumer exposure to caffeine from retail coffee outlets. Food Chem Toxicol 45, 1588-1592.

23. Flint A, Raben A, Blundell JE, et al. (2000) Reproducibility, power and validity of visual analogue scales in assessment of appetite sensations in single test meal studies. Int $J$ Obes Relat Metab Disord 24, 38-48.

24. Astrup A, Buemann B, Western P, et al. (1994) Obesity as an adaption to a high-fat diet: evidence from a cross-sectional study. Am J Clin Nutr 59, 350-355.

25. Bérubé-Parent S, Pelletier C, Doré J, et al. (2005) Effects of encapsulated green tea and guarana extracts containing a mixture of epigallocatechin-3-gallate and caffeine on $24 \mathrm{~h}$ energy expenditure and fat oxidation in men. $\mathrm{Br} J$ Nutr 94, $432-436$
26. Maki KC, Reeves MS, Farmer M, et al. (2009) Green tea catechin consumption enhances exercise-induced abdominal fat loss in overweight and obese adults. J Nutr 139, 264-270.

27. Kajimoto O, Kajimoto Y, Yabune M, et al. (2005) Tea catechins with a galloyl moiety reduce body weight and fat. J Health Sci 51, 161-171.

28. Nagao T, Komine $\mathrm{Y}$, Soga S, et al. (2005) Ingestion of a tea rich in catechins leads to a reduction in body fat and malondialdehydemodified LDL in men. Am J Clin Nutr 81, 122-129.

29. Chantre P \& Lairon D (2002) Recent findings of green tea extract AR25 (Exolise) and its activity for the treatment of obesity. Phytomedicine 9, 3-8.

30. Nagao T, Meguro S, Soga S, et al. (2001) Tea catechins suppress accumulation of body fat in humans. J Oleo Sci $\mathbf{5 0}$, 717-728.

31. Hase T, Komine Y, Meguro S, et al. (2001) Anti-obesity effects of tea catechins in humans. J Oleo Sci 50, 599-605.

32. Tsuchida T, Hiroshige I \& Haruo N (2002) Reduction of body fat in humans by long-term ingestion of catechins. Prog Med 22, 2189-2203.

33. Després J-P \& Lemieux I (2006) Abdominal obesity and metabolic syndrome. Nature 444, 881-887.

34. Diepvens K, Kovacs EMR, Nijs IMT, et al. (2005) Effect of green tea on resting energy expenditure and substrate oxidation during weight loss in overweight females. Br J Nutr 94, $1026-1034$.

35. Kovacs EMR, Lejeune MPGM, Nijs I, et al. (2004) Effects of green tea on weight maintenance after body-weight loss. $\mathrm{Br} \mathrm{J}$ Nutr 91, 431-437.

36. Hsu T-F, Kusumoto A, Abe K, et al. (2006) Polyphenolenriched oolong tea increases fecal lipid excretion. Eur J Clin Nutr 60, 1330-1336.

37. Hill AM, Coates AM, Buckley JD, et al. (2007) Can EGCG reduce abdominal fat in obese subjects? J Am Coll Nutr 26, $396 s-402 s$. 